\title{
Sex differences in fetal growth and immediate birth outcomes in a low-risk Caucasian population
}

Sander Galjaard ${ }^{1,2^{*}}$ (D) Lieveke Ameye ${ }^{3}$, Christoph C. Lees ${ }^{1,4}$, Anne Pexsters ${ }^{1}$, Tom Bourne ${ }^{1,5}$, Dirk Timmerman ${ }^{1}$ and Roland Devlieger ${ }^{1}$

\begin{abstract}
Background: According to the WHO Multicentre Growth Reference Study Group recommendations, boys and girls have different growth trajectories after birth. Our aim was to develop gender-specific fetal growth curves in a lowrisk population and to compare immediate birth outcomes.

Methods: First, second, and third trimester fetal ultrasound examinations were conducted between 2002 and 2012. The data was selected using the following criteria: routine examinations in uncomplicated singleton pregnancies, Caucasian ethnicity, and confirmation of gestational age by a crown-rump length (CRL) measurement in the first trimester. Generalized Additive Model for Location, Scale and Shape (GAMLSS) was used to align the time frames of the longitudinal fetal measurements, corresponding with the methods of the postnatal growth curves of the WHO MGRS Group.

Results: A total of 27,680 complete scans were selected from the astraia $\odot$ ultrasound database representing 12,368 pregnancies. Gender-specific fetal growth curves for biparietal diameter (BPD), head circumference (HC), abdominal circumference $(\mathrm{AC})$, and femur length ( $\mathrm{FL}$ ) were derived. The $\mathrm{HC}$ and BPD were significantly larger in boys compared to girls from 20 weeks of gestation onwards $(p<0.001)$ equating to a 3-day difference at 20-24 weeks. Boys were significantly heavier, longer, and had greater head circumference than girls $(p<0.001)$ at birth. The Apgar score at 1 min $(p=0.01)$ and arterial cord $\mathrm{pH}(p<0.001)$ were lower in boys.

Conclusions: These longitudinal fetal growth curves for the first time allow integration with neonatal and pediatric WHO gender-specific growth curves. Boys exceed head growth halfway of the pregnancy, and immediate birth outcomes are worse in boys than girls. Gender difference in intrauterine growth is sufficiently distinct to have a clinically important effect on fetal weight estimation but also on the second trimester dating. Therefore, these differences might already play a role in early fetal or immediate neonatal management.
\end{abstract}

Keywords: Fetal anthropometric gender differences, Prenatal growth, Birth outcomes, Perinatal and neonatal management

\footnotetext{
* Correspondence: s.galjaard@erasmusmc.nl

${ }^{1} \mathrm{KU}$ Leuven Department of Development and Regeneration: Pregnancy,

Fetus and Neonate, Gynaecology and Obstetrics, University Hospitals Leuven,

Herestraat 49, 3000 Leuven, Belgium

${ }^{2}$ Department of Obstetrics and Gynaecology, Division of Obstetrics and

Prenatal Medicine, Erasmus MC, University Medical Centre, Wytemaweg 80,

3015, CN, Rotterdam, the Netherlands

Full list of author information is available at the end of the article
}

(c) The Author(s). 2019 Open Access This article is distributed under the terms of the Creative Commons Attribution 4.0 International License (http://creativecommons.org/licenses/by/4.0/), which permits unrestricted use, distribution, and reproduction in any medium, provided you give appropriate credit to the original author(s) and the source, provide a link to the Creative Commons license, and indicate if changes were made. The Creative Commons Public Domain Dedication waiver (http://creativecommons.org/publicdomain/zero/1.0/) applies to the data made available in this article, unless otherwise stated. 


\section{Background}

Ultrasound has been an indispensable tool for diagnosis in obstetrics and fetal growth assessment for at least 4 decades [1-3]. Clinical management in pregnancies is increasing based on ultrasound measurements derived in the first trimester and on the recognition of pathological fetal growth, which depends on reliable, standardized growth curves [4]. Although it is widely known that boys are slightly larger than girls in the first trimester and at birth, there has been no consideration of fetal gender in the development and interpretation of fetal growth curves [5-8]. This gender dichotomy seems important since there is clear evidence that gestation-specific neonatal outcomes are worse in boys, indicating the vulnerability of the male embryo and fetus $[9,10]$.

Many charts have been published on fetal growth using different methodologies from the early 1990s until early in this decade, after which new (dating) protocols emerged [11]. Most normal ranges were designed from crosssectional data [12-19], which by their nature may represent fetal size at a given point but do not directly infer growth. To derive information on fetal growth, statistical strategies using repeat measurements are required but longitudinal methodologies are utilized more rarely $[20,21]$. Given these complexities, the World Health Organization (WHO) Multicentre Growth Reference Study (MGRS) Group recommended Generalized Additive Model for Location, Scale and Shape (GAMLSS) for the construction of the WHO Growth Standards [22, 23]. Most recently, growth charts have been developed in the regions of Europe and the USA and customization based on ethnicity is reported $[11,12,18,19,24]$.

Our aim was to develop gender-specific longitudinal first, second, and third trimester normal growth reference curves within a low-risk Caucasian population with a robust WHO-endorsed longitudinal statistical methodology. Further, we aimed to test the validity of these curves by comparing the estimated fetal weights derived from these charts to actual birth weight, and determine whether there were gender differences in fetal growth trajectories and immediate birth outcomes.

\section{Methods}

This was an observational longitudinal cohort study of first, second, and third trimester fetal biometry ultrasound examinations performed during 2002-2012 in the University Hospital Leuven. The study was approved by the ethics committee of the University Hospitals KU Leuven. The data was selected from the astraia $\odot$ ultrasound database with the following criteria (Fig. 1): indication "routine fetal growth" (level 1 and 2 ultrasound scanning for fetal anomalies, excluded), singleton pregnancy, ethnicity "Caucasian," and gestational age confirmed by a crown-rump length (CRL) measurement (3-
$83 \mathrm{~mm}$ ) in the first trimester [25]. Only pregnancies with at least two or maximum three scans (first, second, and third trimester) were selected, representing a routine of care scheme for a low-risk population. The measurements were performed with the following ultrasound machines (with time period of usage): Kretz Voluson 730 (2002-2006), ESAOTE Technos (2002-2006), Acuson Sequoia (2002-2007), General Electric Voluson ${ }^{\circledR} 730$ Expert (GE Healthcare Medical Systems, Kretztechnik, Zipf, Austria, 2007-2012), General Electric Voluson E8 (GE Healthcare Medical Systems, Kretztechnik, Zipf, Austria, 2007-2012). The first three devices were equipped with a $4-8-\mathrm{MHz}$ curved linear array probe. The GE Voluson E730 and GE Voluson E8 used a curved $4-8-\mathrm{MHz}$ volumetric $3 \mathrm{D}$ abdominal probe. All growth data were immediately stored in an electronic database (astraia $\odot$ Software Inc., Munich, Germany). Fetal measurements were based on the following twodimensional biometric parameters: biparietal diameter (BPD), head circumference (HC), abdominal circumference $(\mathrm{AC})$, and femur length (FL), as designated in the guideline descriptions (Additional file 1) [26]. Only the complete fetal datasets (all four measurements) were analyzed. Neonatal data from the included patients were extracted from their birth files for gestational age at delivery, gender, birth weight, birth length, head circumference, Apgar scores (AS) for the first and fifth minute after birth, umbilical cord arterial $\mathrm{pH}$, and base excess (BE) measurement. Only the gender-specific neonatal datasets were analyzed.

\section{Statistical analysis}

Outliers in BPD, $\mathrm{HC}, \mathrm{AC}$, or FL were removed from the data. Generalized Additive Models for Location, Scale and Shape (GAMLSS; www.gamlss.org) was applied to construct the growth curves for all four fetal routine fetal biometry measurements: BPD, HC, AC, and FL, by the use of the $\mathrm{R}$ package software [22, 23]. We assessed several distributions: Box-Cox-t, Box-Cox Cole and Green, and Box-Cox power exponential. Goodness-of-fit of the models was assessed with QQ plots, Akaike Information Criteria (AIC), and worm plots. The goodnessof-fit was investigated covering the gestational age 1240-week period and for substrata of this period. GAMLSS smoothed the antenatal growth curves for $\mathrm{BPD}, \mathrm{HC}, \mathrm{AC}, \mathrm{FL}$, and estimated fetal weight (EFW). For the EFW, the Hadlock-3 formula $\left[\log _{10} \quad E F W=\right.$ 1.3350.0034 (AC) $(\mathrm{FL})+0.0316(\mathrm{BPD})+0.0457(\mathrm{AC})+$ $0.1623(\mathrm{FL})]$ was used [11]. The 5th, 10th, 50th, 90th, and 95th percentiles were plotted with grid lines. The whole analysis was done three times: for all pregnancies, for boys, and for girls. SAS 9.4 was used for merging the fetal database with the neonatal database and analyzing the neonatal data (Mann-Whitney test). 


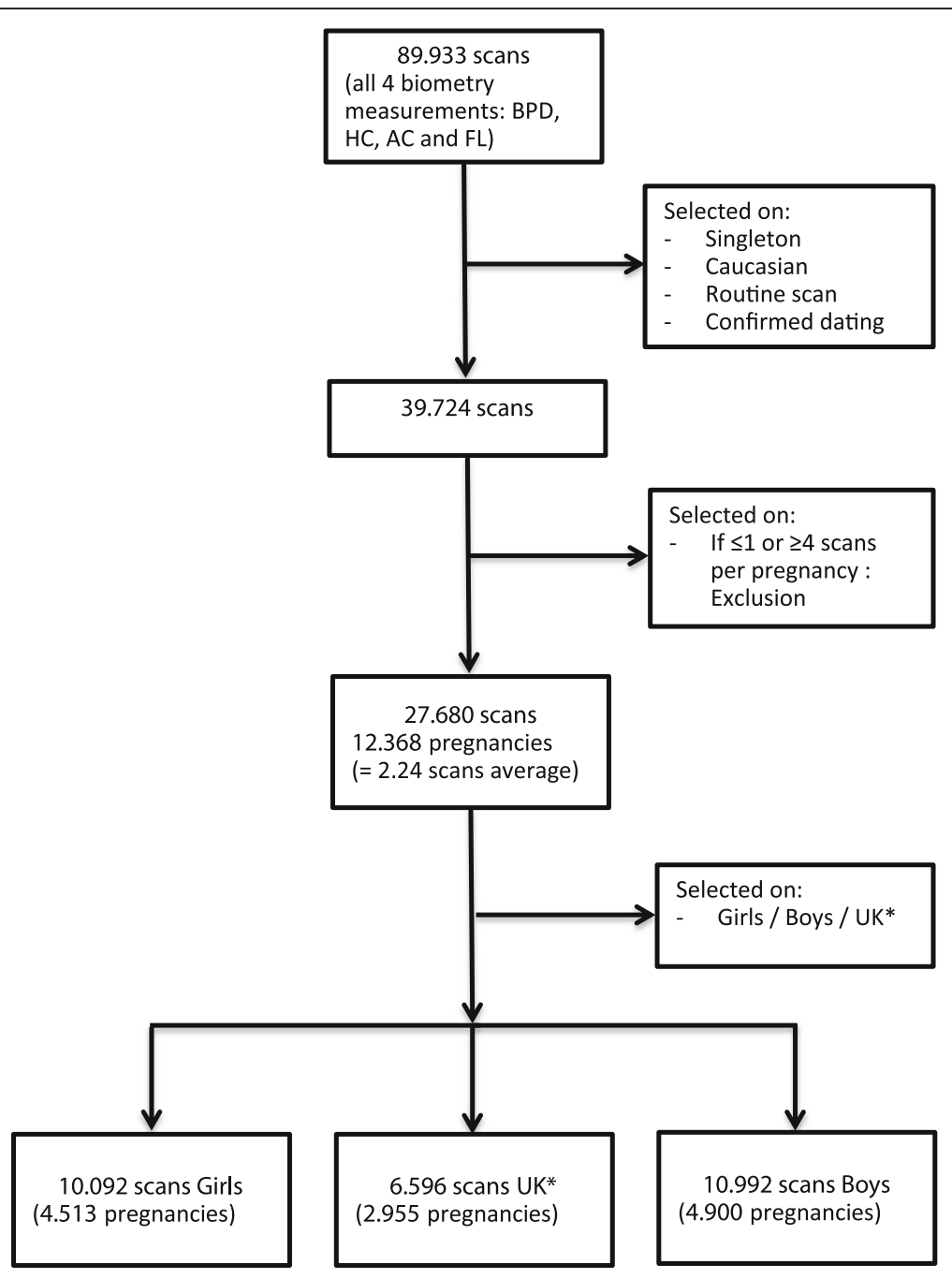

Fig. 1 Flowchart on the selection procedure for normal routine fetal ultrasound scans between 2002 and 2012. *UK, unknown gender

Table 1 Neonatal data for boys, girls, and combined in term and preterm pregnancies

\begin{tabular}{|c|c|c|c|c|}
\hline & Total $(N=9413)$ & Boys $(N=4900,52.1 \%)$ & Girls $(N=4513,47.9 \%)$ & $p$ value $^{*}$ \\
\hline GA at birth (wks, 0/7d), mean \pm std & $39 w k s 2 / 7 \pm 1 w k s 5 d$ & $39 w k s 2 / 7 \pm 1 w k s 5 d$ & $39 w k s 3 / 7 \pm 1 w k s 5 d$ & 0.02 \\
\hline Birth weight $(\mathrm{g})$, mean \pm std & $3392 \pm 512$ & $3450 \pm 515$ & $3329 \pm 502$ & $<0.001$ \\
\hline Length $(\mathrm{cm})$, mean \pm std & $50.5 \pm 2.4$ & $50.9 \pm 2.4$ & $50.1 \pm 2.4$ & $<0.001$ \\
\hline $\mathrm{HC}(\mathrm{cm})$, mean $\pm \mathrm{std}$ & $34.6 \pm 1.6$ & $34.9 \pm 1.6$ & $34.3 \pm 1.5$ & $<0.001$ \\
\hline $1-\min \mathrm{AS} \leq 5$ & $3.4 \%$ & $3.8 \%$ & $2.9 \%$ & 0.01 \\
\hline $5-\min$ AS $\leq 7$ & $2.8 \%$ & $3.2 \%$ & $2.3 \%$ & 0.009 \\
\hline $\mathrm{pH}$ Umb Art, mean \pm std & $7.27 \pm 0.07$ & $7.269 \pm 0.072$ & $7.274 \pm 0.075$ & $<0.001$ \\
\hline $\mathrm{pH}<7.10$, mean \pm std & $1.9 \%(177), 7.04 \pm 0.06$ & $0.9 \%(82), 7.041 \pm 0.059$ & $1.0 \%(95), 7.042 \pm 0.062$ & 0.90 \\
\hline $\mathrm{BE}<-10 \mathrm{mEq} / \mathrm{L}$, mean $\pm \mathrm{std}$ & $1.4 \%(132),-13.46 \pm 4.01$ & $0.8 \%(71),-13.8 \pm 4.2$ & $0.6 \%(61),-13.1 \pm 3.7$ & 0.30 \\
\hline
\end{tabular}

Neonatal demographic data available in 9413 (76\%) of the selected cases "2002-2012." * $p$ value represents difference in boys vs girls

GA gestational age, wks weeks, $d$ days, $g$ gram, $c m$ centimeter, $H C$ head circumference, AS Apgar scores, $p H$ pondus hydrogenium, Umb Art umbilical artery, $B E$ base excess, std standard deviation 
Table 2 Neonatal data for boys, girls, and combined in preterm $(<37$ weeks) pregnancies

\begin{tabular}{|c|c|c|c|c|}
\hline & Total $(N=576)$ & Boys $(N=317,55.0 \%)$ & Girls $(N=259,45.0 \%)$ & $p$ value* \\
\hline GA at birth (wks, 0/7d), mean \pm std & $34 w k s 6 / 7 \pm 2 w k s 2 / 7$ & $35 w k s 0 / 7 \pm 2 w k s 1 / 7$ & $34 w k s 5 / 7 \pm 2 w k s 3 / 7$ & 0.19 \\
\hline Birth weight $(\mathrm{g})$, mean \pm std & $2481 \pm 637$ & $2553 \pm 604$ & $2392 \pm 665$ & 0.003 \\
\hline Length $(\mathrm{cm})$, mean \pm std & $46.3 \pm 4.2$ & $46.8 \pm 4.1$ & $45.7 \pm 4.3$ & 0.005 \\
\hline $\mathrm{HC}(\mathrm{cm})$, mean $\pm \mathrm{std}$ & $32.5 \pm 2.5$ & $32.8 \pm 2.4$ & $32.2 \pm 2.6$ & 0.006 \\
\hline $1-\min A S \leq 5$ & $8.1 \%$ & $8.6 \%$ & $7.4 \%$ & 0.62 \\
\hline $5-\min$ AS $\leq 7$ & $7.9 \%$ & $8.9 \%$ & $6.7 \%$ & 0.32 \\
\hline $\mathrm{pH}$ Umb Art, mean \pm std & $7.29 \pm 0.07$ & $7.285 \pm 0.070$ & $7.289 \pm 0.076$ & 0.50 \\
\hline
\end{tabular}

Neonatal demographic data available in 576 preterm cases "2002-2012." * $p$ value represents difference in boys vs girls GA gestational age, wks weeks $d$ days, $g$ gram, $c m$ centimeter, $H C$ head circumference, AS Apgar scores, $p H$ pondus hydrogenium, Umb Art umbilical artery, std standard deviation

\section{Results}

Between 2002 and 2012, 89,933 scans were selected. After restricting to a low-risk population, a total of 27 , 680 scans remained representing 12,368 pregnancies (Fig. 1). The mean maternal BMI was $23.8 \mathrm{~kg} / \mathrm{m}^{2}$ (std. 4.8), $6.6 \%$ of the women smoked. Gender-specific birth datasets could be ascertained in $76.1 \%$ of the cases and are outlined in Table 1. In total, we had 4900 boys and 4513 girls, representing respectively 10,992 and 10,092 scans. The mean birth weight, birth length, and head circumference were significantly $(p<0.001)$ different for boys $(3450 \mathrm{~g}, 50.9 \mathrm{~cm}, 34.9 \mathrm{~cm})$ as compared to girls $(3329 \mathrm{~g}, 50.1 \mathrm{~cm}, 34.3 \mathrm{~cm})$. A low 1 -min AS $(\leq 5)$ was more common in boys $(3.8 \%)$ as compared to girls (2.9\%) $(p=0.01)$ as was a low 5-min AS $(\leq 7)$ for boys (3.2\%) compared to girls $(2.3 \%$; Table 1$)(p=0.009)$. The arterial umbilical cord $\mathrm{pH}$ was lower in boys compared to girls $(p<0.001)$. There was no difference in asphyxia, defined as a $\mathrm{pH}<7.10$, in boys $(0.9 \%)$ compared to girls $(1.0 \%, p=0.90)$, and abnormal $\mathrm{BE}(<-10 \mathrm{mEq} / \mathrm{L})$ was the same for both sexes. There was no difference in preterm birth (<37 weeks) for girls $(5.7 \%)$ and boys $(6.5 \%$,

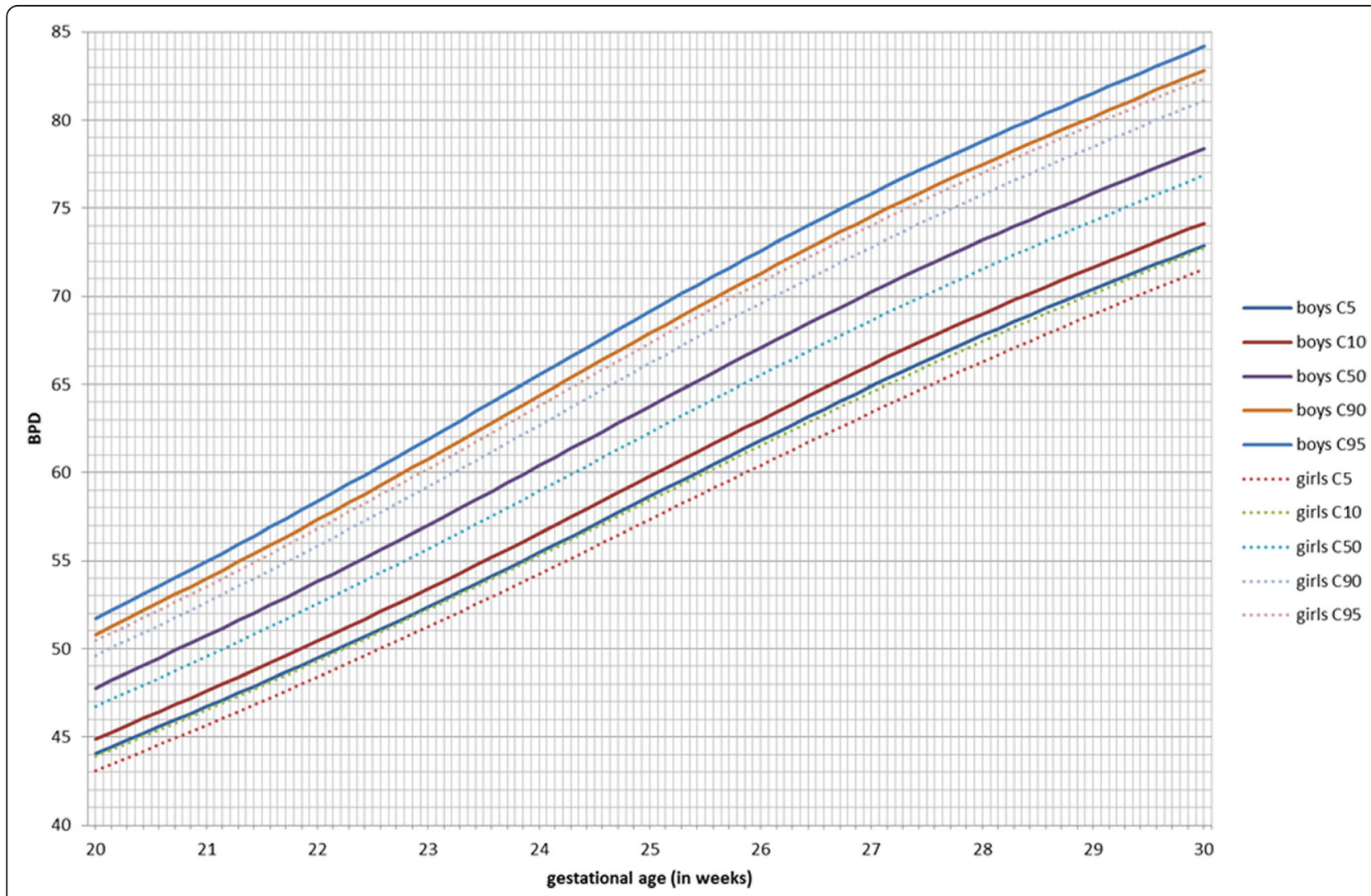

Fig. 2 Biparietal diameter (BPD) in millimeters for boys and girls from 20 to 30 weeks of gestation for percentiles 5, 10, 50, 90, and 95 
$\mathrm{p}=0.14$; Table 2) which occurred in $6 \%$ of the pregnancies overall. In the preterm group, boys were heavier $(p=0.003)$, longer $(p=0.005)$, and had larger head circumferences $(p=0.006)$. The immediate outcome of AS and $\mathrm{pH}$ were also different in boys and girls, although not statistically different due to the smaller preterm group (Table 2). The term group is outlined separately in Additional file 2.

GAMLSS longitudinal fetal antenatal growth curves for BPD, HC, AC, and FL from 12 to 40 weeks were developed for boys, girls, and combined (Additional file 3). For each parameter, the 5th, 10th, 50th, 90th, and 95th centiles were constructed. Actual values for these centiles and grid curves are outlined in Additional file 4. Comparing the two gender growth trajectories and their percentiles, for BPD, there was a significant $(p<0.001)$ difference for all percentiles in boys having higher BPD measurements (Fig. 2, Table 3). At 24 weeks, the 50th percentile BPD for boys $(60.4 \mathrm{~mm})$ is significantly higher as compared to girls $(58.9 \mathrm{~mm}, p<0.001$; Additional file 5). This corresponds to a difference of three gestational days. The boys' 5 th percentile aligns with the 10th percentile of the girls, and the 90th percentile aligns with the 95th percentile of the girls. For $\mathrm{HC}$, these differences were even more pronounced $(p<0.001$; Additional file $5)$. The prenatal difference of $\mathrm{HC}$ of boys at the 95th percentile increases to $+6.5 \mathrm{~mm}$ at 35 weeks, but it is already present at 2 weeks of gestation $(+3.8 \mathrm{~mm}$; Fig. 3 , Table 4). The neonatal head circumference confirmed this difference of $+6 \mathrm{~mm}$ as being significant between boys and girls $(p<0.001$; Table 1$)$. Generally, prenatal AC measurements were significantly higher in boys than in girls, but less demonstrable across the total gestational period than for BPD and HC (Fig. 4). For FL, there was no significant difference between boys and girls in their antenatal growth percentiles (Fig. 5). The EFW was different in boys throughout the gestational age at different percentiles compared to girls, except for the 40 weeks measurement (Table 5). Girls reach the $500 \mathrm{~g}$ EFW 1 day later $(22 \mathrm{wks} 3 / 7)$ as compared to the boys (22wks2/7; Additional file 5). At the 50th percentile at 24 weeks, boys are estimated to be $21 \mathrm{~g}$ heavier compared to girls ( $p=0.02$; Additional file 5).

\section{Discussion}

In this study, we have constructed antenatal growth and estimated fetal weight charts, with a strict and clearly defined selection protocol in a normal Caucasian population and separately for boys and girls. Boys have significantly larger late -second and third trimester $\mathrm{HC}$, $\mathrm{BPD}$, and $\mathrm{AC}$ measurements than girls. For FL, there are no differences. The implication of these findings is that a boy and a girl at exactly 24 weeks of gestation might, based on the current late second trimester dating
Table 3 BPD reference values for boys and girls from 12-40 weeks

\begin{tabular}{|c|c|c|c|c|c|c|c|c|c|c|}
\hline \multirow[t]{2}{*}{$\mathrm{A}$} & \multicolumn{5}{|c|}{ Boys (BPD) } & \multicolumn{5}{|c|}{ Girls (BPD) } \\
\hline & $\mathrm{C} 5$ & $\mathrm{C} 10$ & 50 & 90 & 5 & $\mathrm{C} 5$ & $\mathrm{C} 10$ & $\mathrm{C} 50$ & C90 & 95 \\
\hline & 16.7 & 7.3 & 19.4 & 21.6 & 3 & 16.5 & 17.1 & 19.2 & 21.3 & 219 \\
\hline & 20.4 & 21.0 & 23.2 & 25.6 & 26.3 & 20.1 & 7 & 22.9 & 25.1 & 25.7 \\
\hline & 2 & 25.0 & 27.3 & 29.7 & 30.4 & 24.0 & 24.6 & 26.8 & 29.1 & 29.7 \\
\hline & 282 & 80 & 31.3 & 338 & 345 & 278 & 28.5 & 30.8 & 33.1 & 337 \\
\hline & 32 & 32.6 & & 37.6 & & & 1 & 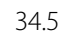 & 36.8 & 37.5 \\
\hline & 35.3 & 36.0 & 38 & 41.0 & 418 & 7 & 35.4 & 379 & 4 & 41.1 \\
\hline & 38.3 & 3 & 4 & 4 & & 8 & $3 \varepsilon$ & 41.0 & 4 & \\
\hline & 41.3 & 4 & 4 & 47.6 & & .5 & 41.3 & 4.0 & 4 & 47.5 \\
\hline & 44.0 & 44.9 & 4 & 50.8 & & & 4 & 46.7 & & \\
\hline & 46.7 & 47.6 & 50.7 & 54. & 55 & 45.7 & 46.5 & 49.6 & 52 & 53.5 \\
\hline & 49.5 & 50.4 & 5 & 57.3 & 584 & 48.4 & 49.3 & 5 & 5 & 568 \\
\hline & 52.4 & 53.4 & 57 & 60.8 & & 5 & 52.2 & 55.7 & 5 & \\
\hline & 55 & 56.6 & 60.4 & 64.3 & 65.5 & 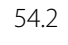 & 5 & 9 & 62.7 & 638 \\
\hline & 58. & 5 & 63 & 6 & & & 58.4 & 6 & & \\
\hline & 61.8 & 63.0 & 67 & 71. & 72.6 & 604 & 61.5 & 655 & 60 & 708 \\
\hline & 04.9 & 6 & 70 & 74.5 & $1 / 0.0$ & 63.4 & 64.6 & 6 & 72.8 & 14.0 \\
\hline & 67.8 & 69. & 73. & 77.5 & 78.8 & 66.3 & 67.4 & 71.5 & 75 & 1.0 \\
\hline & 70.4 & 71.6 & 75.8 & 80.2 & & & 70.2 & 74.3 & 78.5 & 707 \\
\hline & 72.9 & 74.1 & 78.4 & 82.8 & 84.2 & 71.6 & 72.7 & 76.9 & 81.1 & 82.4 \\
\hline & 75 & 76.6 & 81 & 85.5 & & 74.0 & 75 & 78 & 8 & \\
\hline & 77. & 7 & 83.3 & 87.9 & & 76.2 & 77.4 & 81.7 & 86.0 & 87.3 \\
\hline & 795 & 80.9 & 854 & 90.1 & 916 & 8.1 & 79.4 & 83 & 887 & 80 \\
\hline & 01 & ${ }_{0}$ & 0 & 92.1 & & & 8 & o & & \\
\hline & 82.5 & 84.0 & 889 & 939 & 055 & 813 & 82.7 & 87.3 & 921 & 935 \\
\hline & 83.6 & 85.1 & 90.3 & o. & & & 84.1 & 88.9 & 93.9 & \\
\hline & 84.6 & 86.2 & 91.7 & 97.4 & 9 & 84.1 & 85.5 & 90.5 & 95.6 & 97.2 \\
\hline & (6. & 87.4 & 然 & נ. & 201? & 855 & 87.0 & 2 & 97.4 & . \\
\hline & 87.0 & 88.8 & 94.9 & 101.2 & 3.3 & 86.9 & 88.4 & 93.7 & 99.3 & 100 \\
\hline & . & ( & ro. & 103.2 & (10.0. & 88.2 & 89.8 & נת.J & 101.1 & 10 \\
\hline
\end{tabular}

Reference values in millimeters for fetal biparietal diameter (BPD) for boys and girls for each gestational week for the median and 5th, 10th, 90th, and 95th centiles GA gestational age

protocols with head measurements, be assigned a gestation as much as 3-day difference and an EFW difference of $21 \mathrm{~g}$ at 24 weeks favoring the boys. These antenatal differences were confirmed at birth with boys being significantly heavier, longer, and having larger head circumferences as compared to girls. The 1- and 5-min AS and cord $\mathrm{pH}$ was lower in boys. The dating and weight estimation differences could potentially be taken into account in determining prenatal and immediate perinatal viability management in terms of timing the administration of maternal steroids for fetal lung maturation, decisions for delivery, and possible resuscitation. Also, in the post-term period management in pregnancy, these 


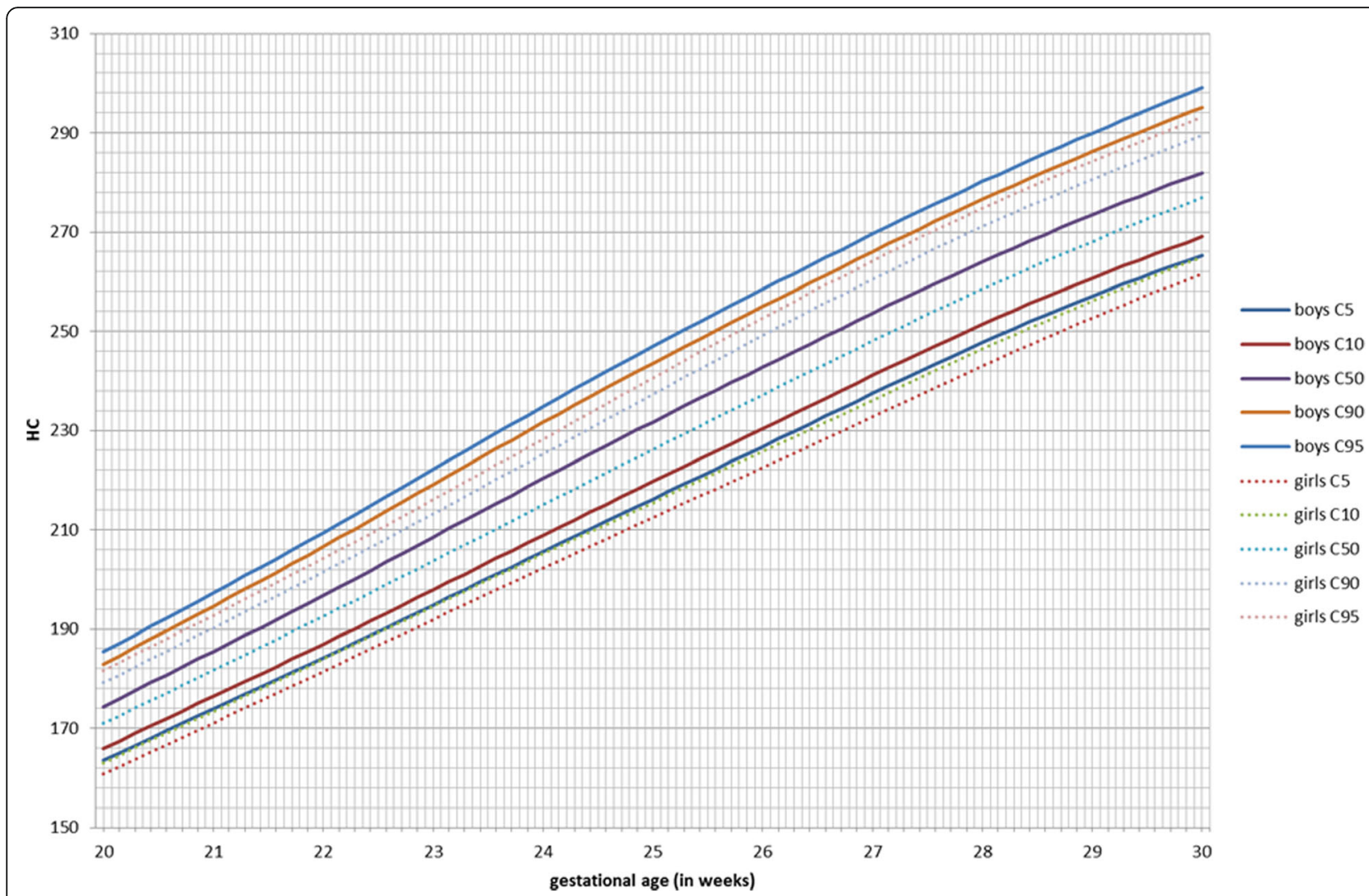

Fig. 3 Head circumference (HC) in millimeters for boys and girls from 20 to 30 weeks of gestation for percentiles 5, 10, 50, 90, and 95

gender differences could also potentially influence decisions including the timing of labor inductions, affecting an even larger population. Consequently, if second trimester dating of the pregnancy has been undertaken, girls are potentially put at risk of stillbirth in the postterm period by assuming the gestational maturity to be less than it is [27].

In one cross-sectional study, a difference has been shown between fetal head measurements for both boys and girls, although the curves were constructed with the older linear regression models [28]. They also confirmed the birth weight difference but did not report information on neonatal head circumference or other outcomes (AS, cord pH). Another unselected multi-ethnic combined cross-sectional and longitudinal population study also found differences in fetal head and abdomen measurements using statistical methods current at that time; however, no birth outcomes were available [29].

While it has been demonstrated that gestation-specific neonatal outcomes are worse in boys than in girls [9, $10]$, what had not been previously appreciated in a routine population is that boys have lower Apgar scores at both 1 and $5 \mathrm{~min}$ and lower cord $\mathrm{pH}$ values at delivery than girls. These results underline male vulnerability in the perinatal period. In a recent published elegant report on neonatal outcome in appropriately grown term babies, gender differences were demonstrated in terms of lower Apgar scores at $5 \mathrm{~min}$ and higher rates of instrumental deliveries for failure to progress in labor for boys [30]. This concerned a multi-ethnic retrospective cohort from one center and birth data specified for both genders. They demonstrated a birth weight difference of $135 \mathrm{~g}$ at term, comparing closely with the $121 \mathrm{~g}$ that we report, but their data lacked other anthropometric data (birth length and head circumference) and antenatal growth data. It is of course possible that neonatal outcomes are worse because immediate birth outcomes are worse. Whether this is an attribute of being male per se, or some effect of fetal size on delivery, cannot be explained from their results or ours. We can demonstrate that the gender differences in fetal anthropometry starting from 20 weeks onwards affect fetal dating and the estimated fetal weight. In our preterm sub-analysis, the birth weight differences between boys and girls are also present in absolute mean differences ( $\Delta$ birth weight 161 g, $\Delta$ birth length $0.8 \mathrm{~cm}, \Delta \mathrm{HC} 0.6 \mathrm{~cm}$ ), and there are noticeable differences between AS and umbilical cord $\mathrm{pH}$ (Table 2), although not statistically significant due to smaller numbers. One hypothesis is that either the differences in biometry are relatively more important in the 
Table $4 \mathrm{HC}$ reference values for boys and girls from 12 to 40 weeks

\begin{tabular}{|c|c|c|c|c|c|c|c|c|c|c|}
\hline \multirow[t]{2}{*}{$\overline{G A}$} & \multicolumn{5}{|c|}{ Boys (HC) } & \multicolumn{5}{|c|}{ Girls (HC) } \\
\hline & $\mathrm{C} 5$ & $\mathrm{C} 10$ & C50 & C90 & C95 & C5 & C10 & C50 & C90 & C95 \\
\hline 12 & 61.3 & 63.3 & 70.3 & 77.4 & 79.4 & 61.9 & 63.6 & 69.7 & 76.1 & 78.1 \\
\hline 13 & 74.4 & 76.5 & 83.8 & 91.1 & 93.2 & 74.3 & 76.1 & 82.6 & 89.5 & 91.6 \\
\hline 14 & 88.2 & 90.3 & 97.8 & 105.3 & 107.5 & 87.3 & 89.2 & 96.1 & 103.3 & 105.4 \\
\hline 15 & 101.9 & 104.1 & 111.6 & 119.2 & 121.4 & 100.6 & 102.6 & 109.6 & 117.0 & 119.2 \\
\hline 16 & 115.2 & 117.4 & 124.9 & 132.6 & 134.8 & 113.6 & 115.6 & 122.8 & 130.4 & 132.6 \\
\hline 17 & 128.0 & 130.2 & 137.8 & 145.6 & 147.8 & 126.3 & 128.3 & 135.7 & 143.3 & 145.6 \\
\hline 18 & 140.3 & 142.5 & 150.3 & 158.2 & 160.5 & 138.5 & 140.7 & 148.2 & 156.0 & 158.3 \\
\hline 19 & 152.2 & 154.5 & 162.6 & 170.7 & 173.1 & 150.1 & 152.3 & 160.0 & 168.0 & 170.4 \\
\hline 20 & 163.4 & 165.8 & 174.3 & 182.9 & 185.4 & 160.7 & 163.0 & 171.0 & 179.2 & 181.6 \\
\hline 21 & 173.9 & 176.4 & 185.5 & 194.6 & 197.3 & 171.1 & 173.4 & 181.7 & 190.2 & 192.8 \\
\hline 22 & 184.2 & 187.0 & 196.8 & 206.6 & 209.5 & 181.5 & 183.9 & 192.6 & 201.5 & 204.2 \\
\hline 23 & 194.8 & 197.9 & 208.5 & 219.1 & 222.2 & 191.9 & 194.5 & 203.7 & 213.2 & 216.0 \\
\hline 24 & 205.6 & 208.9 & 220.3 & 231.6 & 234.8 & 202.3 & 205.1 & 215.0 & 225.2 & 228.3 \\
\hline 25 & 216.2 & 219.7 & 231.7 & 243.6 & 247.0 & 212.5 & 215.5 & 226.2 & 237.3 & 240.6 \\
\hline 26 & 226.8 & 230.4 & 242.8 & 255.0 & 258.5 & 222.5 & 225.8 & 237.2 & 249.1 & 252.7 \\
\hline 27 & 237.4 & 241.1 & 253.6 & 266.0 & 269.6 & 232.7 & 236.1 & 248.0 & 260.5 & 264.2 \\
\hline 28 & 247.7 & 251.4 & 264.0 & 276.5 & 280.2 & 243.0 & 246.4 & 258.5 & 271.1 & 274.8 \\
\hline 29 & 257.1 & 260.7 & 273.4 & 286.2 & 290.0 & 252.7 & 256.1 & 268.1 & 280.6 & 284.3 \\
\hline 30 & 265.3 & 269.0 & 282.0 & 295.1 & 299.0 & 261.6 & 265.0 & 277.0 & 289.5 & 293.2 \\
\hline 31 & 273.0 & 276.8 & 290.0 & 303.7 & 307.7 & 269.6 & 273.1 & 285.5 & 298.3 & 302.1 \\
\hline 32 & 280.2 & 284.0 & 297.6 & 311.9 & 316.3 & 276.5 & 280.2 & 293.2 & 306.7 & 310.8 \\
\hline 33 & 286.5 & 290.4 & 304.4 & 319.4 & 324.1 & 282.6 & 286.4 & 300.1 & 314.4 & 318.7 \\
\hline 34 & 292.1 & 296.1 & 310.3 & 326.0 & 331.0 & 288.0 & 292.0 & 306.2 & 321.1 & 325.5 \\
\hline 35 & 297.7 & 301.6 & 315.9 & 332.2 & 337.5 & 292.6 & 296.7 & 311.3 & 326.5 & 331.0 \\
\hline 36 & 302.9 & 306.8 & 321.2 & 338.2 & 343.9 & 296.6 & 300.7 & 315.5 & 330.9 & 335.5 \\
\hline 37 & 307.3 & 311.3 & 326.1 & 344.1 & 350.4 & 301.0 & 305.2 & 320.1 & 335.8 & 340.4 \\
\hline 38 & 311.9 & 316.0 & 331.1 & 350.4 & 357.5 & 306.5 & 310.7 & 325.9 & 341.7 & 346.4 \\
\hline 39 & 317.3 & 321.4 & 336.7 & 357.1 & 365.2 & 312.8 & 317.1 & 332.4 & 348.5 & 353.3 \\
\hline 40 & 323.5 & 327.6 & 342.9 & 364.2 & 373.1 & 319.4 & 323.7 & 339.2 & 355.4 & 360.2 \\
\hline
\end{tabular}

Reference values in millimeters for fetal head circumference (HC) for boys and girls for each gestational week for the median and 5th, 10th, 90th, and 95th centiles GA gestational age

(full-grown) male fetus interacting with maternal pelvic limitations causing more labor dystocia for boys, and hence lower AS. Alternatively, other fetal gender-specific factors can influence the birth process and compromising the immediate birth outcomes. Gender-specific body composition at birth has been reported, where the male infant has more fat mass and lean body mass than the female infant, especially in well-nourished mothers [31]. This phenomenon has been associated with genderdifferent intrauterine physical adaptations to an enhanced nutrient supply from the mother. The male infant body composition has been more subject to maternal influences as higher pre-gestational BMI and excessive gestational weight gain [32]. Lastly, the lung maturation of the male fetus proceeds slower than in the female fetus, possibly contributing to a higher rate of low AS in the term grown fetus. In animal studies, lung fluid secretion is inhibited and the lung fluid absorption initiated by adrenalin infusions at birth [33]. And preterm asphyxiated male infants have lower adrenaline levels than female infants, again putting the boys at higher risk [34]. Whether in the term infant this will be similar is unknown.

\section{Strength and weakness}

Our antenatal growth curves are unique in that all four fetal growth parameters (BPD, $\mathrm{HC}, \mathrm{AC}$, and $\mathrm{FL}$ ) were 


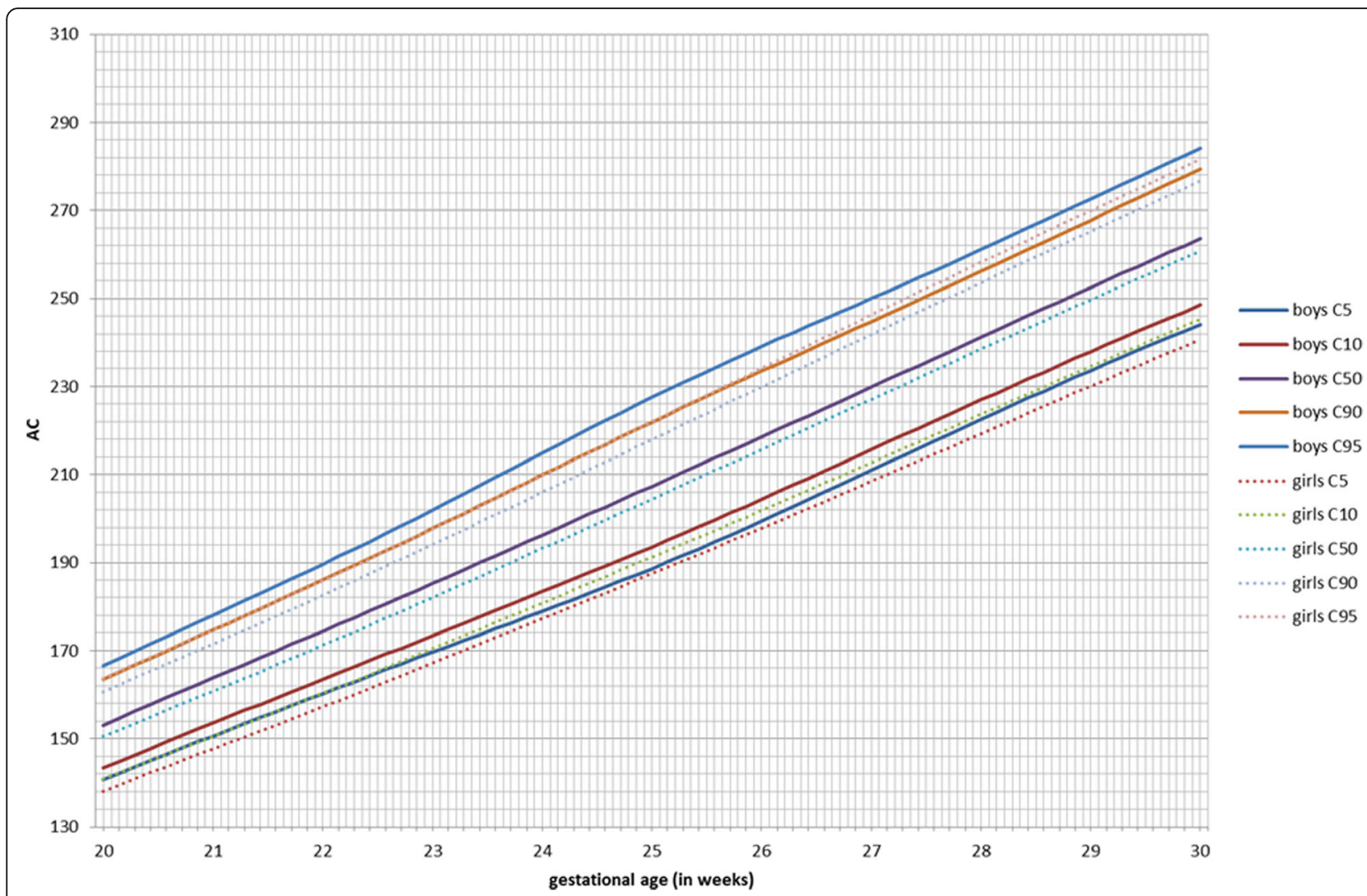

Fig. 4 Abdominal circumference (AC) in millimeters for boys and girls from 20 to 30 weeks of gestation for percentiles 5, 10, 50, 90, and 95

measured in standardized circumstances in accordance with international guidelines [26]. Longitudinal growth charts were constructed for each parameter, with the WHO advocated GAMLSS method used [22, 23]. GAMLSS can combine longitudinal data with a crosssectional component and can construct centiles in a way that they are constrained and do not cross. Further, in using the GAMLSS analysis statistics, one could, by synchronizing the statistical methods of the $\mathrm{WHO}$, align the biometry measurements with the neonatal and pediatric charts [22, 23]. With the available neonatal data, we could discriminate different growth curves for boys and girls for all four fetal growth parameters and hence the EFW. Since the introduction of ultrasound in antenatal care, many reports on fetal growth curves have been published [11-21]. Recognizing pathological fetal growth depends on reliable, standardized growth curves [35]. Discrepancies between the curves have often been attributed to the differences in methodology and population selection [36]. A recent report reviewed fetal growth charts, demonstrating the wide variations of methodologies on how these charts have been constructed concluding that there were many grounds for bias in the growth curves that are currently used [37]. Particularly in "inclusion/exclusion criteria," "ultrasound quality control measures," and "gestational dating protocols," many ambiguities existed. Standardization of the methodologies with a checklist was recommended to define a highquality study [37]. When we compare our growth charts to the requirements, these would be compliant for the combination of a high-quality control score, longitudinal design, sample size, and the fact that all four parameters (BPD, HC, $\mathrm{AC}$, and FL) were examined (Additional file 6). All growth measurements were reviewed by certified staff members, judging all the scanned images as to whether they adhered to the protocol described. We also incorporated a strict protocol on pregnancy dating. Only pregnancies that had a first trimester confirmation scan on gestational age were included: crown-rump length (CRL) measurement between 3 and $83 \mathrm{~mm}$ (gestational age $\geq 5^{+0}$ and $<14^{+0}$ weeks) $[4,25]$. In Belgium, in routine obstetrical care, every pregnant woman will be offered a first, second, and third trimester ultrasound scan with fetal growth measurements. In many countries, the third trimester scan is not part of the routine care for low-risk pregnancies [38]. Also, to measure the four fetal growth parameters in the first trimester is not a routine care and allowed us to define "fetal growth" through serial measurements, instead of "fetal size," as 


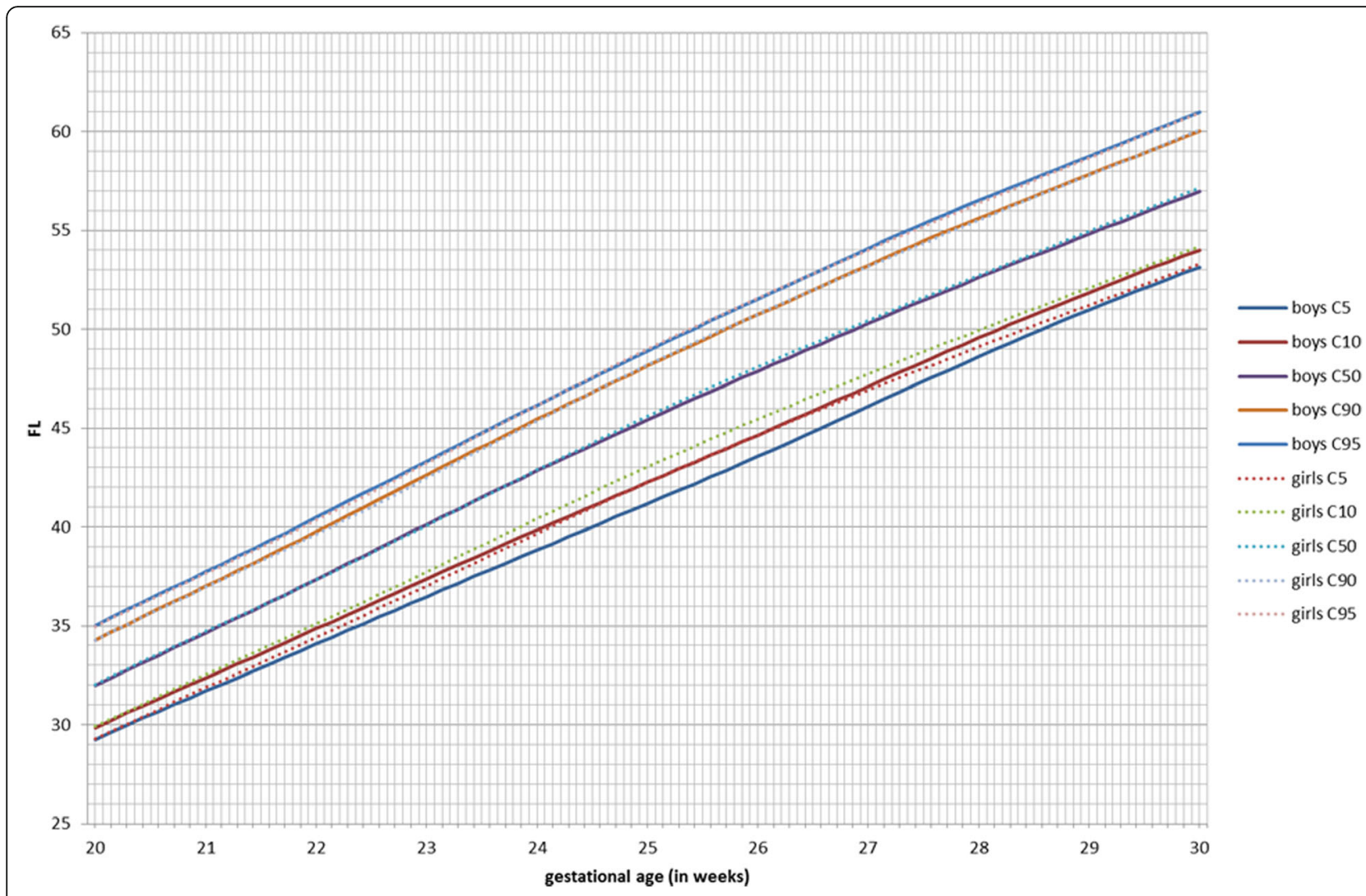

Fig. 5 Femur length (FL) in millimeters for boys and girls from 20 to 30 weeks of gestation for percentiles 5, 10, 50, 90, and 95

defined through cross-sectional measurements [12-19, 39]. Furthermore, we were able to eliminate aberrant fetal growth and extreme maternal influences by excluding fetal anomalies (level 1 and 2 indications) and including only the mothers enrolled to a routine obstetric care scheme [40]. Finally, a population-based cohort was generated with a significant sample size over a period of 11 years. The description of a routine population could also be supported by our neonatal data. Neonatal data was complete for $76 \%$ in our cohort. The rate of premature birth was $6 \%$, which is consistent with the European nationally accepted norms. In our population selection, we further customized the charts for one maternal and one fetal factor. We selected on ethnicity "Caucasian" and the fetal gender. Other ethnicityderived customized growth curves have arisen in response to the early reference charts from mainly Europe and the USA [18, 19]. Ethnicity was reported to have a discriminative influence on fetal growth [24, 41]. The aim of the INTERGROWTH-21st study was to construct prescriptive instead of descriptive curves using the same statistical methods as used in our study (GAMLLS) [42]. The study population comprised $35 \%$ of the pregnant population, recruited highly selected healthy, educated ( $>75 \%$ of a local level), non-obese (BMI $18-30 \mathrm{~kg} / \mathrm{m}^{2}$ ), non-smoking women, 18-35 years of age and recruited in selected institutes. This highly qualitative study (Additional file 6) represents a fascinating investigation of the physiology of fetal growth, concluding that optimal growth potential can be attained irrespective of the ethnicity in a selected population, which is in contradiction with the previous studies. Unfortunately, it lacks information on fetal gender differences; not all measurements were longitudinal, and the derived charts are by their selective nature manifestly not representative of a general population, regardless of the ethnicity concerned. Our current study adds these advantages. Girls and boys both have different neonatal growth curves, assuming there is a discriminative effect of the gender on their growth trajectories. In more than three quarters of our cohort, complete neonatal data was registered, including gender registration. Therefore, we focused on developing two separate fetal growth charts, both for boys and girls. Comparing the extremes of growth $(<\mathrm{p} 5$ and $>$ p95), the female fetus is considered wrongfully small or non-macrosomic and the male fetus vice versa when compared to the INTERGROWTH-21st curves (Table 6). Fetal gender, unlike maternal ethnicity, is not commonly known in the first trimester but it is from the 20 weeks' scan onwards ("anomaly" scan). From a clinical point of view, it seemed therefore relevant to start discriminating these curves from 20 weeks of gestation onwards. 
Table 5 EFW reference values for boys and girls from 12 to 40 weeks

\begin{tabular}{|c|c|c|c|c|c|c|c|c|c|c|}
\hline \multirow[t]{2}{*}{ GA } & \multicolumn{5}{|c|}{ oys (EFW) } & \multicolumn{5}{|c|}{ Girls (EF } \\
\hline & $\mathrm{C} 5$ & 10 & 0 & & 5 & C5 & $\mathrm{C} 10$ & 50 & C90 & 95 \\
\hline & 50 & 51 & 57 & 63 & 65 & & & & & 63 \\
\hline & 60 & & & & & & & & & 79 \\
\hline & & 80 & & & & & & & & \\
\hline & 104 & 107 & 120 & 136 & & & & & & \\
\hline & & & & & & & & & 65 & \\
\hline & 159 & 165 & 185 & 208 & & 159 & 164 & 183 & 206 & 21 \\
\hline & 199 & & & & & & & & & \\
\hline & 248 & 256 & 287 & 324 & 336 & 242 & 250 & 28 & 316 & 328 \\
\hline & 200 & 308 & & & & & & & & \\
\hline & 352 & 36 & & & & & & & 4 & 4 \\
\hline & 414 & & 486 & & & & & & & \\
\hline & 488 & 50 & 5 & 6 & & & & & & \\
\hline & 574 & 596 & 681 & 781 & & 561 & 582 & 660 & & (27) \\
\hline & & 698 & & & & & & & & \\
\hline & . & 810 & 0 & 1 & 111 & 7 & 795 & 905 & 1032 & 107 \\
\hline & 901 & 938 & 1073 & & & 883 & & & & \\
\hline & 103 & 108 & 12 & 1 & 14 & 10 & 10 & & & \\
\hline & 1188 & 1235 & 1407 & & & & & & & \\
\hline & 1348 & 140 & 15 & 18 & & & 13 & & & \\
\hline & 1511 & 15 & 17 & 2035 & 2116 & 1478 & 1536 & & & \\
\hline & & & & & & & & & & \\
\hline & 1825 & 1 & 2 & 2 & 2 & $\beta$ & 1863 & 2128 & 2437 & 254 \\
\hline & 1970 & 2 & 2 & & & & & & & \\
\hline & 2108 & 2309 & 2 & 3 & 2 & 50 & 56 & 00 & 12 & 3059 \\
\hline & 2244 & 235 & & & & & & & & \\
\hline & 2379 & 2506 & 2974 & 3 & 0 & 2 & 24 & 2887 & 3428 & \\
\hline & 2513 & 2655 & 3174 & 3795 & 400 & 2475 & 2610 & 3095 & 3707 & \\
\hline & 264 & 280 & 3 & 0 & & 2626 & 4 & 3310 & 3999 & \\
\hline & & 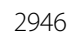 & & $452 J$ & 4591 & 2700 & $29+5$ & זכנת & T500 & 4605 \\
\hline
\end{tabular}

Reference values in grams for estimated fetal weight (EFW) for boys and girls for each gestational week for the median and 5th, 10th, 90th, and 95th centiles

$G A$ gestational age

Some limitations on constructing these charts have to be addressed. The study was performed in a university teaching hospital, a large tertiary referral center, not necessarily reflecting a routine setting. This center, on the other hand, also has a regional remit for routine obstetric care for low-risk pregnancies, but the included cases were not selected on maternal morbidity nor on parental characteristics. Some maternal characteristics (e.g., smoking occurred in 6.6\%) were not excluded in the selected cohort, deliberately to prevent "supernormalization" of the cohort. But artificial conception was excluded for intracytoplasmic sperm injection, since
Table 6 Cross-sectional gestational age comparison of INTERGROWTH-21st and gender-specific (M/F) fetal head measurements at 5 th and 95 th percentiles

\begin{tabular}{lllllllll}
\hline GA & C5 & IG & F & M & C95 & IG & $F$ & M \\
\hline BPD & & & & & & & & \\
14 & 24.2 & 26.8 & 24.0 & 24.3 & 30.2 & 32.5 & 29.7 & 30.4 \\
15 & 28.2 & 29.6 & 27.8 & 28.2 & 34.5 & 35.6 & 33.7 & 34.5 \\
16 & 31.5 & 32.5 & 31.4 & 32.0 & 38.0 & 38.8 & 37.5 & 38.3 \\
20 & 43.5 & 44.7 & 43.1 & 44.0 & 51.3 & 52.2 & 50.5 & 51.7 \\
24 & 54.7 & 57.0 & 54.2 & 55.5 & 64.8 & 65.7 & 63.8 & 65.5 \\
30 & 72.2 & 73.9 & 71.6 & 72.9 & 83.6 & 84.0 & 82.4 & 84.2 \\
34 & 80.6 & 82.4 & 79.8 & 81.2 & 92.9 & 93.4 & 91.6 & 93.7 \\
37 & 84.7 & 86.7 & 84.1 & 84.6 & 98.2 & 98.4 & 97.2 & 99.2 \\
HC & & & & & & & & \\
14 & 87.6 & 88.7 & 87.3 & 88.2 & 108.0 & 107.1 & 105.4 & 107.5 \\
15 & 101.8 & 100.6 & 100.6 & 101.9 & 123.3 & 120.1 & 119.2 & 121.4 \\
16 & 113.9 & 112.6 & 113.6 & 115.2 & 135.3 & 133.2 & 132.6 & 134.8 \\
20 & 161.8 & 160.2 & 160.7 & 163.4 & 184.4 & 184.7 & 181.6 & 185.4 \\
24 & 203,9 & 205.4 & 202.3 & 205.6 & 233.3 & 232.7 & 228.3 & 234.8 \\
30 & 263.4 & 263.2 & 261.6 & 265.3 & 297.2 & 293.6 & 293.2 & 299.0 \\
34 & 290.2 & 291.5 & 288.0 & 292.1 & 328.9 & 324.7 & 325.5 & 331.0 \\
37 & 302.9 & 305.7 & 301.0 & 307.3 & 346.0 & 342.7 & 340.4 & 350.4 \\
\hline
\end{tabular}

Reference values in millimeters for fetal biparietal diameter (BPD) and head circumference $(\mathrm{HC})$ for gestational landmark weeks for the 5th and 95th centiles. Total group (C5 and C95), INTERGROWTH-21st (IG) and fetal gender current study (F/M)

$G A$ gestational age (in weeks)

this is a level 1 ultrasound indication. Finally, it is expected that within this large time period, some women with subsequent pregnancies were included more than once for this cohort.

\section{Implications for clinical practice}

Our fetal growth curves for the Caucasian population resemble predictive growth curves with the gender specified which can discern aberrant from normal fetal growth. The longitudinal aspect and large cohort, covering the full trimesters, have not been reported before in the Caucasian population. The neonatal data gave us the opportunity to customize for the fetal gender. There was a marked difference between fetal boys and girls in their growth trajectory for fetal head measurements and to a lesser extent the abdominal circumferences. Also for the estimated fetal weight, there was a difference. This gender differentiation is important in antenatal and perinatal care. Prenatal ultrasound is used not only to define fetal growth, but also gestational age. Both growth and fetal age are important in defining the time point of fetal viability and the optimization of the timing of obstetrical interventions, e.g., medical elective birth or administration of corticosteroids for fetal lung maturation in cases 
of threatened premature birth. Second trimester dating depends on fetal growth parameters and particular on the fetal head measurement. Our results suggest a gender-specific approach in counseling future parents on important issues when fetal viability starts and when is the best time point to start obstetrical interventions.

The gender differences are further demonstrated by the immediate birth outcomes for males: different anthropometry (heavier, longer, and bigger heads), lower $\mathrm{AS}$, and lower cord $\mathrm{pH}$. The significant lower AS and umbilical cord $\mathrm{pH}$ in boys underline the fetal male vulnerability, although in the asphyxia group $(\mathrm{pH}<7.10)$, there was no predominance by males, stating that boys do not have a higher risk of acidemia at birth in a routine population. Therefore, one can argue on the clinical importance of the $\mathrm{pH}$ findings (and perhaps also the AS) in our study.

\section{Conclusion}

In summary, we present fetal growth curves with the latest statistical tools in a large, routine pregnant population with state-of-the-art ultrasound technology. The data covers the pregnancy period from 12 weeks onwards, and there were differences between boys and girls for the fetal head and fetal abdomen measurements and the estimated fetal weight. Also, the immediate neonatal outcome demonstrated gender differences favoring the girls. This could give caretakers the opportunity to take into account a gender-tailored approach in life decision care both at the margins of viability and post-term.

\section{Additional files}

Additional file 1: Ultrasound protocol guidelines [26]. (DOCX $12 \mathrm{~kb}$ )

Additional file 2: Table S1. Neonatal data for boys, girls and combined in term ( $\geq 37$ weeks) pregnancies. (DOCX $23 \mathrm{~kb}$ )

Additional file 3: Boys vs Girls combined scatterplots and curves. (DOCX $816 \mathrm{~kb}$ )

Additional file 4: Boys vs Girls combined gridcurves and reference values. (DOCX $4051 \mathrm{~kb}$ )

Additional file 5: Boys vs Girls combined centiles and real values. (DOCX $35 \mathrm{~kb}$ )

Additional file 6: Table S2. Fetal charts characteristics, quality control. (DOCX $17 \mathrm{~kb})$

\section{Acknowledgements}

Not applicable.

\section{Authors' contributions}

SG, RDV, and AP conceived and designed the study, with additional support from $L A, C C L, T B$, and DT. SG and $L A$ were involved in the data cleaning. $L A$ and SG analyzed the data, with support from CCL, TB, DT, and RDV. SG, LA, $\mathrm{CCL}$, and RDV were involved in the data interpretation. SG, LA, CCL, and RDV wrote the first draft of the manuscript, which was then critically reviewed and revised by the other co-authors. All authors approved the final version of the manuscript for submission. All authors had full access to all of the data (including statistical reports and tables) in the study and can take responsibility for the integrity of the data and the accuracy of the data analysis. SG, CCL, DT, and RDV are the guarantors.

\section{Funding}

TB and CCL are supported by the National Institute for Health Research (NIHR) Biomedical Research Centre based at Imperial College Healthcare NHS Trust and Imperial College London. DT and RDV are Senior Clinical Investigators of the Research Foundation Flanders (FWO). The views expressed are those of the authors and not necessarily those of the NHS, NIHR, or Department of Health. The sponsors had no role in study design; in the collection, analysis, and interpretation of the data; in the writing of the report; and in the decision to submit the work for publication. The researchers performed this work independently of the funding sources.

\section{Availability of data and materials}

The datasets used and/or analyzed during the current study are available from the corresponding author on reasonable request.

\section{Ethics approval and consent to participate}

The research was approved by the ethics committee of the University Hospitals KU Leuven.

Consent for publication

Not applicable.

\section{Competing interests}

All authors declare that they have no competing interests.

\section{Author details}

${ }^{1} \mathrm{KU}$ Leuven Department of Development and Regeneration: Pregnancy, Fetus and Neonate, Gynaecology and Obstetrics, University Hospitals Leuven, Herestraat 49, 3000 Leuven, Belgium. ${ }^{2}$ Department of Obstetrics and Gynaecology, Division of Obstetrics and Prenatal Medicine, Erasmus MC, University Medical Centre, Wytemaweg 80, 3015, CN, Rotterdam, the Netherlands. ${ }^{3}$ Department of Development and Regeneration, KU Leuven, Herestraat 49, 3000 Leuven, Belgium. ${ }^{4}$ Centre for Fetal Care, Queen Charlotte's and Chelsea Hospital, Imperial College Healthcare NHS Trust, Imperial College London, London, UK. ${ }^{5}$ Department of Gynaecology and Obstetrics, Queen Charlotte's and Chelsea Hospital, Imperial College Healthcare NHS Trust, Imperial College London, London, UK.

Received: 28 May 2019 Accepted: 26 August 2019

Published online: 09 September 2019

\section{References}

1. Donald I, McVicar J, Brown T. Investigations of abdominal masses by pulsed ultrasound. Lancet. 1958;1(7032):1188-95.

2. Campbell S. An improved method of fetal cephalometry by ultrasound. J Obstet Gynaecol Br Commonw. 1968:75:568-76.

3. Campbell $\mathrm{S}$. The prediction of fetal maturity by ultrasonic measurement of the biparietal diameter. J Obstet Gynaecol Br Commonw. 1969;76:603-9.

4. Napolitano R, Dhami J, Ohuma EO, et al. Pregnancy dating by fetal crownrump length: a systematic review of charts. BJOG. 2014. https://doi.org/1 $0.1111 / 1471-0528.12478$

5. Lary JM, Paulozzi LJ. Sex differences in the prevalence of human birth defects: a population-based study. Teratology. 2001;64:237-51.

6. Rogers JF, Graves WL. Risk factors associated with low Apgar scores in a low-income population. Paediatr Perinat Epidemiol. 1993;7:205-16.

7. Bekedam DJ, Engelsbel S, Mol BWJ, et al. Male predominance in fetal distress during labor. Am J Obstet Gynecol. 2002;187:1605-7.

8. Bukowski R, Smith GCS, Malone FD, et al. Human sexual size dimorphism in early pregnancy. Am J Epidemiol. 2007;165:1216-8.

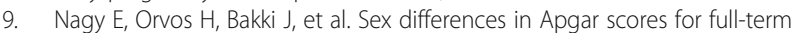
neonates. Acta Paediatrica. 2009;98:897-900.

10. Vatten $L$, Skjaerven R. Offspring sex and pregnancy outcome by length of gestation. Early Human Development. 2004;76:47-54.

11. Hadlock FP, Harrist RB, Sharman RS, et al. Estimation of fetal weight with the use of head, body and femur measurements - a prospective study. Am J Obstet Gynecol. 1985;151:333-7.

12. Snijders RJM, Nicolaides KH. Fetal biometry at 14-40 weeks' gestation. Ultrasound Obstet Gynecol. 1994;4:34-48. 
13. Chitty LS, Altman DG, Henderson A, et al. Charts of fetal size: 2. Head measurements. Br J Obstet Gynaecol. 1994;101:35-43.

14. Chitty LS, Altman DG, Henderson A, et al. Charts of fetal size: 3. Abdominal measurements. Br J Obstet Gynaecol. 1994;101:125-31.

15. Chitty LS, Altman DG, Henderson A, et al. Charts of fetal size: 4. Femur length. Br J Obstet Gynaecol. 1994;101:132-5.

16. Kurmanavicius J, Wright EM, Royston $P$, et al. Fetal ultrasound biometry: 1 . Head reference values. Br J Obstet Gynaecol. 1999:106:126-35.

17. Kurmanavicius J, Wright EM, Royston $\mathrm{P}$, et al. Fetal ultrasound biometry: 2. Abdomen and femur length reference values. Br J Obstet Gynaecol. 1999; 106:136-43.

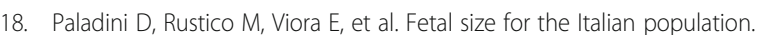
Normative curves of head, abdomen and long bones. Prenat Diagn. 2005; 25:456-64.

19. Leung TN, Pang MW, Daljit SS, et al. Fetal biometry in ethnic Chinese: biparietal diameter, head circumference, abdominal circumference and femur length. Ultrasound Obstet Gynecol. 2008;31:321-7.

20. Johnsen SL, Wilsgaard T, Rasmussen S, et al. Longitudinal reference ranges for estimated fetal weight. Acta Obstet Gynecol Scand. 2006;85:286-97.

21. Verburg BO, Steegers EA, De Ridder M, et al. New charts for ultrasound dating of pregnancy and assessment of fetal growth: longitudinal data from a population-based cohort study. Ultrasound Obstet Gynecol. 2008;31:388-96.

22. Rigby RA, Stasinopoulos DM. Generalized Additive Models for Location, Scale and Shape. Appl Statist. 2005;54:507-54.

23. Borghi $\mathrm{E}$, de Onis M, Garza C, for the WHO Multicentre Growth Reference Study Group, et al. Construction of the World Health Organization child growth standards: selection of methods for attained growth curves. Statist Med. 2006;25:247-65.

24. Merialdi M, Widmer M, Gülmezoglu AM, et al. WHO Multicenter Study for the development of growth standards from fetal life to childhood: the fetal component. BMC Pregnancy and Childbirth. 2014;14:157.

25. Pexsters A, Daemen A, Bottomley $C$, et al. New crown-rump length curve based on over 3500 pregnancies. Ultrasound Obstet Gynecol. 2010;35(6):650-5.

26. ISUOG Prenatal Ultrasound Screening Taskforce. Practice guidelines for performance of the routine mid-trimester fetal ultrasound scan. http://www. isuog.org/NR/rdonlyres/EA865840-6CA3-45AC-9E99-FBAF775119A9/0/ PrenatalUS_GL_Final.pdf. Accessed 14 Sept 2010.

27. Skalkidou A, Kieler H, Stephansson O, et al. Ultrasound pregnancy dating leads to biased perinatal morbidity and neonatal mortality among postterm-born girls. Epidemiology. 2010;21:791-6.

28. Melamed N, Meizner I, Mashiach R, et al. Fetal sex and intrauterine growth patterns. J Ultrasound Med. 2013;32:35-43.

29. Schwarzler P, Bland JM, Holden D, et al. Sex-specific ante-natal reference growth charts for uncomplicated singleton pregnancies at 15-40 weeks' gestation. Ultrasound Obstet Gynecol. 2004;23:23-9.

30. Dunn L, Prior T, Greer R, et al. Gender specific intrapartum and neonatal outcomes for term babies. Eur J Biol Gynecol \& Reprod Biol. 2015;185:19-22.

31. Lampl M, Gotsch F, Kusanovic JP, et al. Sex differences in fetal growth responses to maternal height and weight. Am J Hum Biol. 2010;22:431-43.

32. OTierney-Ginn P, Presley L, Mimium J, et al. Sex-specific effects of maternal anthropometrics on body composition at birth. Am J Obstet Gynecol. 2014; 211:292.e1-9.

33. Olver RE, Walters DV. The effect of catecholamines on foetal lung liquid secretion. J Physiol. 1977;273:58-9.

34. Greenough A, Lagercrantz H, Pool J, et al. Plasma catecholamine levels in preterm infants. Effect of birth asphyxia and Apgar score. Acta Paediatr Scand. 1987;76:54-9

35. Altman DG, Chitty LS. Design and analysis of studies to derive charts of fetal size. Opinion. Ultrasound Obstet Gynecol. 1993;3:378-84.

36. Rasmussen S. Charts to assess fetal wellbeing. Opinion. Ultrasound Obstet Gynecol. 2011;37:2-5

37. Ioannou C, Talbot K, Ohuma E, et al. Systematic review of methodology used in ultrasound studies aimed at creating charts of fetal size. BJOG. 2012; 119:1425-39.

38. Skrastad RB, Eik-Ness SH, Sviggum O, et al. A randomized controlled trial of third-trimester routine ultrasound in a non-selected population. Acta Obstet Gynecol Scand. 2013;92:1353-60.

39. Royston P, Altman DG. Design and analysis of longitudinal studies of fetal size. Opinion. Ultrasound Obstet Gynecol. 1995;6:307-12.
40. Hussain U, Daemen A, Missfelder-Lobos H, et al. Umbilical artery pulsatility index and fetal abdominal circumference in isolated gastroschisis. Ultrasound Obstet Gynecol. 2011;38:538-42.

41. Unterscheider J, Geary PM, Daly S, et al. The customized fetal growth potential: a standard for Ireland. Eur J Obstet Gynecol Reprod Biol. 2013;166:14-7.

42. Papageorghiou AT, Ohuma EO, Altman DG, et al. International standards for fetal growth based on serial ultrasound measurements: the Fetal Growth Longitudinal Study of the INTERGROWTH-21 ${ }^{\text {st }}$ Project. Lancet. 2014;384:869-79.

\section{Publisher's Note}

Springer Nature remains neutral with regard to jurisdictional claims in published maps and institutional affiliations.
Ready to submit your research? Choose BMC and benefit from:

- fast, convenient online submission

- thorough peer review by experienced researchers in your field

- rapid publication on acceptance

- support for research data, including large and complex data types

- gold Open Access which fosters wider collaboration and increased citations

- maximum visibility for your research: over $100 \mathrm{M}$ website views per year

At $\mathrm{BMC}$, research is always in progress.

Learn more biomedcentral.com/submissions 